\title{
Genomics and computational science for virus research
}

\author{
Hironori Sato ${ }^{1 *}$, Masaru Yokoyama ${ }^{1}$ and Hiroyuki Toh ${ }^{2}$ \\ 1 Pathogen Genomics Center, National Institute of Infectious Diseases, Tokyo, Japan \\ ${ }^{2}$ Computational Biology Research Center, National Institute of Advanced Industrial Science and Technology, Tokyo, Japan \\ *Correspondence: hirosato@nih.go.jp \\ Edited by: \\ Akio Adachi, The University of Tokushima Graduate School, Japan \\ Reviewed by: \\ Akio Adachi, The University of Tokushima Graduate School, Japan
}

RNA viruses are highly mutable, yet changes in genomes and proteins would be restricted by the functional and structural constraints inherent in the survival strategies of viruses in nature. Rapidly evolving technologies in genomics and computational science are now opening up a new avenue for elucidating the real picture of diversity of the organism in nature and for studying the principles underlying the maintenance and change of structures, interactions, and functions of biomolecules. The information is essential for understanding the evolutionary dynamics of virus-host interactions in virological, immunological, and epidemiological phenomena and for rationally developing methods to control RNA viruses. In this Research Topic, we present 17 timely articles, consisting of 5 reviews, 3 mini reviews, 7 original researches, 1 hypothesis \& theory, and 1 perspective, all of which underscore the challenges and increasing importance of incorporating the new technologies to study RNA viruses and their impacts on hosts.

\section{EXPLORATIONS OF VIRAL OUASISPECIES, MICRORNAs, AND ANTIBODYOMES IN NATURE}

Beerenwinkel et al. (2012) reviewed the challenges and opportunities in inferring the diversity of intra-host virus populations using next-generation sequencing technologies. They discuss the wisdom of reducing artificial errors during sample preparation, existing approaches inferring local and global diversity from sequence data, and successful applications on basic and biomedical studies. Tan Gana et al. (2012) reviewed the latest articles describing cellular and viral microRNAs involved in HIV-1 infection. They describe recent advances in understanding of the biogenesis and functions of the microRNAs in the virus-cell battles and point out roles of the genomics and computational science in obtaining and integrating the information.

Prabakaran et al. (2012) reported on the antibodyomes of 10 healthy individuals obtained by 454 pyrosequencing and bioinformatics analyses. They showed genetic evidence that the antibody subsets with distinct diversity and related to the already-known neutralizing antibodies against the HIV-1, SARS coronavirus, and henipaviruses exist in human IgM repertoires of uninfected individuals. Zhu et al. (2012) reported an antibodyome of an HIV-1-infected individual who produced broadly neutralizing antibodies. Using 454 pyrosequencing, bioinformatics, and functional analyses, they suggested a role of somatic maturation in generating heavy- and light-chain sequences with varied neutralization phenotypes against HIV-1.

\section{COMPUTATIONAL ANALYSES OF THE 3-D STRUCTURES, INTERACTIONS, AND EVOLUTION OF PROTEINS USING GENETIC INFORMATION}

Ode et al. (2012) reviewed the results of molecular dynamic simulations to learn the structural dynamics of proteins in solution. They highlight studies on the structure and function of viral enzymes, virion structures, mechanisms of viral resistance against host immunities and anti-viral drugs, and the development of anti-viral agents. Franzosa et al. (2012) reviewed structural systems biology of interactomes in the host-pathogen relationships. They present existing experimental datasets of the host-pathogen interactome and discuss approaches to obtain structural interactome by integrating the biophysical, functional, and evolutionary information. Miki and Katayama (2012) presented a viewpoint on the in silico 3-D structural analysis in virus research. They describe importance of incorporating in silico modeling techniques into experimental studies to solve structural problems in their neutralization study of the norovirus.

Bozek et al. (2012) provided in silico structural models of capsid proteins of HIV-2 and SIV, which revealed marked differences in the electrostatic potential on the interaction surface and suggested a potential role of electrostatic interactions in the evasion of SIV from the rhesus restriction factor Trim5a. Daiyasu et al. (2012) reported a new application of information theory to the study of the divergent evolution of function of chemokine receptors and their homologs, such as decoy and viral receptors, in which both sequence and structural information are used to identify amino acid positions that might be responsible for evolving their distinct functions. Rusu et al. (2012) provided in silico structural models of the Rift Valley fever virus glycoproteins Gn and Gc. The models with the cryo-electron microscopy data allowed the authors to identify four possible arrangements of the glycoproteins in the virion envelope and to indicate how these proteins assemble to form the capsomer base and intercapsomer connections. Yokoyama et al. (2012) provided in silico structural models of sapovirus protease docked to its substrate peptides; these models described how this enzyme realizes the functional binding of cleavage sites with distinct sequences and allowed rational identification of the sapovirus protease inhibitors in combination with experimental approaches.

\section{ANALYSES OF VIRUS-CELL INTERACTIONS, VIRAL REPLICATION, AND HOST IMMUNE RESPONSES}

Iwami et al. (2012) reported a mathematical model to quantitatively characterize the viral replication in cell cultures. In their 
study, the data from two time-course experiments of infections with a cell-free virus stock are used to estimate the half-life of infected cells, viral production rate of an infected cell, and the basic reproductive number. Takemura and Murakami (2012) reviewed structure and function of HIV-1 capsid proteins. They describe the capsid structure in relation to their abilities to form a conical core in a virion or to interact with various cellular proteins that promote or suppress viral replication. Nomura and Matano (2012) reviewed the critical roles of host HLA/MHC-I genotypes in disease progression in primate lentivirus infections. They highlighted studies showing the association of the HLA/MHC-I genotypes with rapid or slow AIDS progression during HIV/SIV persistent infections. Kuroki et al. (2012) reviewed the structural biology of the immunologically intriguing cell surface receptors termed paired receptors. By referencing recent studies of two major structural superfamilies, the immunoglobulin-like and the C-type lectin-like receptors, they described how these receptors discriminate self and non-self ligands to maintain homeostasis in the immune system.

\section{REFERENCES}

Beerenwinkel, N., Gunthard, H. F., Roth, V., and Metzner, K. J. (2012). Challenges and opportunities in estimating viral genetic diversity from next-generation sequencing data. Front. Microbiol. 3:329. doi: 10.3389/fmicb.2012.00329

Bozek, K., Nakayama, E. E., Kono, K., and Shioda, T. (2012). Electrostatic potential of human immunodeficiency virus type 2 and rhesus macaque simian immunodeficiency virus capsid proteins. Front. Microbiol. 3:206. doi: 10.3389/ fmicb.2012.00206

Daiyasu, H., Nemoto, W., and Toh, H. (2012). Evolutionary analysis of functional divergence among chemokine receptors, decoy receptors, and viral receptors. Front. Microbiol. 3:264. doi: 10.3389/ fmicb.2012.00264

Franzosa, E. A., Garamszegi, S., and Xia, Y. (2012). Toward a three-dimensional view of protein networks between species. Front. Microbiol. 3:428. doi: 10.3389/ fmicb.2012.00428

Iwami, S., Sato, K., De Boer, R. J., Aihara, K., Miura, T., and Koyanagi, Y. (2012). Identifying viral parameters from in vitro cell cultures. Front. Microbiol. 3:319. doi: 10.3389/fmicb.2012.00319

Kuroki, K., Furukawa, A., and Maenaka, K. (2012). Molecular recognition of paired receptors in the immune system. Front. Microbiol. 3:429. doi: 10.3389/ fmicb.2012.00429

Miki, M., and Katayama, K. (2012). In silico 3D structure analysis accelerates the solution of a real viral structure and antibodies docking mechanism. Front. Microbiol. 3:387. doi: 10.3389/fmicb.2012.00387

Nomura, T., and Matano, T. (2012). Association of MHC-I genotypes with disease progression in HIV/SIV infections. Front. Microbiol. 3:234. doi: 10.3389/fmicb.2012.00234

Ode, H., Nakashima, M., Kitamura, S., Sugiura, W., and Sato, H. (2012). Molecular dynamics simulation in virus research. Front. Microbiol. 3:258. doi: 10.3389/ fmicb.2012.00258

Prabakaran, P., Zhu, Z., Chen, W., Gong, R., Feng, Y., Streaker, E., et al. (2012). Origin, diversity, and maturation of human antiviral antibodies analyzed by high-throughput sequencing. Front. Microbiol. 3:277. doi: 10.3389/fmicb. 2012.00277

\section{ANALYSES OF VIRAL TRANSMISSION NETWORK, EVOLUTION, AND RE-EMERGING DISEASES}

Shiino (2012) reviewed phylodynamic analyses of viral infections, transmission, and evolution in host populations. The author highlighted recent viral researches inferring epidemiologically important parameters, such as infectious clusters, transmission connections, basic reproductive number, and fluctuation of the viral population size. Sasaki et al. (2012) proposed a mathematical model that describes the temporal dynamics of the spread of poliovirus avirulent and virulent strains in a human population; this model was used to estimate the risk of outbreak following the cessation of administration of oral polio vaccine and to predict conditions that significantly increased the outbreak risk.

In summary, these articles present overviews and examples of "Frontiers in Virology" to elucidate viral diversity, protein 3-D structures, virus-host interactions, and evolution of RNA viruses using forefront technologies in genomics and computational science.

Rusu, M., Bonneau, R., Holbrook, M. R., Watowich, S. J., Birmanns, S., Wriggers, W., et al. (2012). An assembly model of rift valley Fever virus. Front. Microbiol. 3:254. doi: 10.3389/fmicb.2012.00254

Sasaki, A., Haraguchi, Y., and Yoshida, H. (2012). Estimating the risk of re-emergence after stopping polio vaccination. Front. Microbiol. 3:178 doi: $10.3389 /$ fmicb. 2012.00178

Shiino, T. (2012). Phylodynamic analysis of a viral infection network. Front. Microbiol. 3:278. doi: $10.3389 /$ fmicb. 2012.00278

Takemura, T., and Murakami, T. (2012). Functional constraints on HIV-1 capsid: their impacts on the viral immune escape potency. Front. Microbiol. 3:369. doi: 10.3389/ fmicb.2012.00369

Tan Gana, N. H., Onuki, T., Victoriano, A. F., and Okamoto, T. (2012). MicroRNAs in HIV-1 infection: an integration of viral and cellular interaction at the genomic level. Front. Microbiol. 3:306. doi: 10.3389/fmicb.2012.00306

Yokoyama, M., Oka, T., Kojima, H., Nagano, T., Okabe, T., Katayama, K., et al. (2012). Structural basis for specific recognition of substrates by sapovirus protease.
Front. Microbiol. 3:312. doi: 10.3389/fmicb.2012.00312

Zhu, J., O’Dell, S., Ofek, G., Pancera, M., Wu, X., Zhang, B., et al. (2012). Somatic populations of PGT135137 HIV-1-neutralizing antibodies identified by 454 pyrosequencing and bioinformatics. Front. Microbiol. 3:315. doi: 10.3389/ fmicb.2012.00315

Received: 18 February 2013; accepted: 18 February 2013; published online: 07 March 2013

Citation: Sato H, Yokoyama $M$ and Toh H (2013) Genomics and computational science for virus research. Front. Microbiol. 4:42. doi: 10.3389/ fmicb.2013.00042

This article was submitted to Frontiers in Virology, a specialty of Frontiers in Microbiology.

Copyright () 2013 Sato, Yokoyama and Toh. This is an open-access article distributed under the terms of the Creative Commons Attribution License, which permits use, distribution and reproduction in other forums, provided the original authors and source are credited and subject to any copyright notices concerning any third-party graphics etc. 